\title{
Historia moderna de un Hospital y de la Cirugía en Regiones
}

\author{
Modern history of a Hospital and Surgery of Regions
}

\author{
Mauricio Cáceres P. ${ }^{1}$
}

En 1980, el Hospital Base San José de Osorno pasa a formar parte del Servicio de Salud Osorno convirtiéndose en el único Hospital de Alta Complejidad de la Provincia. En esa época se desempeño como Jefe del Servicio de Urgencia y luego Jefe de Cirugía por 13 años el Dr Gustavo Perez Nylund quien fue uno de los primeros especialistas en cirugía general procedente formado en el Hospital J.J.A. Le correspondió desarrollar fructíferamente la cirugía digestiva y torácica e implementar la colecistectomia laparoscopica, retirándose con más de 4 décadas de abnegado y destacado servicio público, el que ha seguido cumpliendo en la Clínica Alemana y en la Dirección del nuevo Centro de Diagnostico y Referencia de la Municipalidad de Osorno.

El paso de los años y el crecimiento de la población fue haciendo a este Hospital insuficiente, por lo que en los años 90 comenzó a desarrollarse el Proyecto de Normalización para satisfacer las necesidades futuras, en el que le correspondió trabajar junto a otras Jefaturas, al Dr. Daniel Lilayu Vivanco, cirujano posbecario del Hospital San Borja Arriaran, en su calidad de nuevo Jefe de Urgencia; luego como Jefe de Cirugía, por 2 periodos. Gracias a su visión y amistad con el Profesor y Director del Instituto de Cirugía de entonces, Dr. Aliro Venturelli Leonelli , se fortalecieron los vínculos académicos con la Facultad de Medicina de la Universidad Austral de Chile (UACH), comenzando las rotaciones de Internos de cirugía por urgencias y luego en la formación de nuestros propios alumnos de Medicina. Dicho médico se destacó profesionalmente en la cirugía vascular periférica y en la constitución de los actuales equipos quirúrgicos en las principales especialidades, para finalmente dejar la Cirugía canalizando su vocación de servicio público, primero como Concejal y en la actualidad como Gobernador Provincial de Osorno.

Durante esa época se desempeñó como Director del Servicio de Salud de Osorno, el connotado psiquiatra, Dr. Mauricio Jeldrez, en cuya administración se concretaron muchos avances en la salud pública y las dos primeras misiones de estudio para las especialidades derivadas de cirugía plástica y reparadora y cirugía coloproctológica en la Universidad de Chile.

Volviendo a la modernización de la infraestructura, el Pergamino de Construcción del actual Hospital normalizado se firmó el 13 de septiembre del 2007 y contempló que se ubicara en el mismo lugar, aprovechando gran parte de las instalaciones remodeladas y construyendo $20 \mathrm{mil} \mathrm{m}^{2}$ de nuevas edificaciones, entregándose la primera parte a la comunidad en marzo de 2010, con una torre de 5 pisos diseñada con los más altos estándares de seguridad, nuevo Servicio de Urgencia con sala de reanimación, observación, cirugía menor, yeso y otro sector de camas críticas intercomunicadas con 18 cupos de adultos, dotado de 11 pabellones quirúrgicos, uno de los cuales fue equipado con tecnología que permite transmitir operaciones en tiempo real, gracias a un proyecto para el pos grado, financiado por el Ministerio de Salud, además de una unidad de cirugía mayor ambulatoria con 14 cupos y entrada independiente. El segundo nivel de la Torre lo constituye un piso mecánico con instalaciones de apoyo y administrativas. En los pisos superiores están los servicios clínicos quirúrgicos, medicina interna, oncología y pensionado, ginecología y obstetricia y pediatría, con oficinas administrativas y auditorio equipado en cada piso.

En el edificio administrativo se construyó un auditorio central para 200 personas, equipado con tecnología multimedia y que se utiliza permanentemente en capacitación y educación médica y a la comunidad.

En el Consultorio Adosado de Especialidades se construyeron amplias salas de espera separadas de las consultas y con puertas diferenciadas para no entorpecer la circulación interna.
'Presidente Capítulo X-XIXIII Regiones. Miembro del Directorio Sociedad de Cirujanos de Chile.

Correspondencia a: mcaceres1960@gmail.com 
Los servicios de apoyo fueron instalados en $11.000 \mathrm{~m}^{2}$ remodelados: imagenología, farmacia, hemodiálisis, medicina física y rehabilitación, laboratorio, anatomía patológica, endoscopía con sala de desinfección de alto nivel, esterilización, banco de sangre, alimentación, servicios generales y movilización.

Toda esta gran modernización permitió lograr el $1^{\circ}$ de Febrero del 2010 la calidad de Establecimiento Auto gestionado en Red.

Lo anterior, sumado a la impronta de las nuevas autoridades y jefaturas, ha permitido continuar con el perfeccionamiento y especialización médica, completando 14 cirujanos en cirugía general 12 en especialidades derivadas de cabeza y cuello, vascular periférico, cirugía digestiva, coloproctología y de tórax conformándose los equipos quirúrgicos necesarios, para brindar un Servicio de la mas alta calidad y seguridad.

Otro hito importante fue en febrero de 2016, al convertirse en el primer Hospital de Alta Complejidad, en la X Región en cumplir con el 100\% de las características obligatorias del Estándar General de Acreditación para prestadores institucionales de atención cerrada y con un $84,8 \%$ de cumplimiento del total de las características evaluadas en dicho procedimiento, permitiendo constituirse como un prestador GES.

Este constante progreso facilitó que pudiera ser formalizado el Convenio Docente Asistencial con la Facultad de Medicina de la UACH, el que fue renovado hasta el año 2026, certificándonos ante Asofamech como campo clínico de pre grado manteniendo más de 100 alumnos, dictando 40 asignaturas con 23 docentes en planta académica y otros tantos profesores adjuntos, permitiendo a la fecha, el egreso de 104 médicos en 10 promociones y la acreditación de la carrera de Medicina por 7 años. A esto le hemos sumado nuestra presentación ante APICE como centro de formación y practica de pos grado, permitiendo las rotaciones de becarios UACH de traumatología, a los que sumamos de cirugía, con 22 a la fecha; ginecología-obstetricia; psiquiatría; medicina familiar y finalmente urología, con un promedio de 15 por año, proyectándose el inicio de neurología. En cuanto a infraestructura señalamos la incorporación al edificio de la Facultad, que ya quedó estrecho, de un sitio de $2.400 \mathrm{~m}^{2}$, con casa habitación para residencia de becarios, salas de estudio y administrativas, sumándose a lo anterior el proyecto de construcción de 2 edificios institucionales más, de 5 pisos c/u con $2.267 \mathrm{~m}^{2}$ para estudios Interdisciplinarios, de pos grado

A lo anterior, tenemos que sumar el Convenio de Cooperación Internacional firmado con la Universidad Medico Dental de Tokio y la entrada en vigencia el año 2017 del Convenio de Intercambio Académico con la Universidad Católica de Lovaina que ha permitido realizar pasantías a 30 docentes, entre ellos 4 cirujanos, en sub especialidades del Hospital Saint Luc de Bruselas, Bélgica.

La historia de la cirugía de Osorno se ha desarrollado de la mano con la historia del hospital y de la UACH y el futuro está por escribirse, pero es prometedor, toda vez que ya han asumido la cada vez más compleja gestión clínica, las nuevas generaciones de entusiastas y preparados cirujanos, dispuestos a cargar con el peso de la historia, las contingencias del presente y el desafío tecnológico del futuro. 